\title{
Isoimperatorin induces apoptosis of the SGC-7901 human gastric cancer cell line via the mitochondria-mediated pathway
}

\author{
KEHUI TONG ${ }^{1}$, CHANG XIN $^{2}$ and WENZHONG CHEN ${ }^{3}$ \\ Departments of ${ }^{1}$ Oncological Surgery, ${ }^{2}$ Hepatobiliary Surgery and ${ }^{3}$ General Surgery, Yinzhou People's Hospital, \\ School of Medicine, Ningbo University, Ningbo, Zhejiang 315040, P.R. China
}

Received July 3, 2015; Accepted October 25, 2016

DOI: $10.3892 / \mathrm{ol} .2016 .5387$

\begin{abstract}
The present study was designed to investigate the antiproliferative activity of isoimperatorin against SGC-7901 cells and to examine the possible mechanisms. The antiproliferative activity of isoimperatorin against SGC-7901 cells was evaluated using an MTT assay, and the mechanisms were investigated using flow cytometry and western blot assays, which were used to determine the apoptotic rate and expression levels of mitochondria-mediated apoptosis-associated proteins, including Survivin, myeloid leukemia cell-1 (Mcl-1), B cell lymphoma-extra large (Bcl-xl), B cell lymphoma 2 (Bcl-2), second mitochondria-derived activator of caspase (Smac), Bcl-2-associated X factor (Bax), cleaved (c)-caspase-3 and c-caspase-9 in SGC-7901 cells. Additionally, a xenograft assay was used to confirm whether isoimperatorin had an inhibitory effect on SGC-7901 cell-induced tumors in vivo. The results of the MTT assay suggested that isoimperatorin significantly inhibited the proliferation of SGC-7901 cells in a dose- and time-dependent manner, and the half maximal inhibitory concentration was $18.75 \mu \mathrm{g} / \mathrm{ml}$. The results of the flow cytometric analysis indicated that, following treatment with isoimperatorin, the apoptotic rate of SGC-7901 cells was significantly increased, compared with that of cells in the control group. The results of the western blot analysis indicated that, following treatment with isoimperatorin, the expression levels of the pro-apoptotic proteins, Bax, c-caspase-3 and c-caspase- 9 , were significantly increased and the expression levels of the anti-apoptotic proteins, Survivin and Bcl-2, were significantly reduced, compared with the control group. No alterations in expression were found in the other apoptosis-associated proteins, including Mcl-1, Bcl-xl and Smac. The results of the xenograft assay indicated that
\end{abstract}

Correspondence to: Mr. Wenzhong Chen, Department of General Surgery, Yinzhou People's Hospital, School of Medicine, Ningbo University, 251 Baizhang East Road, Ningbo, Zhejiang 315040, P.R. China

E-mail:wzcheninnb@163.com

Key words: isoimperatorin, SGC-7901 cells, proliferation, mitochondria-mediated apoptosis, in vitro, in vivo isoimperatorin significantly inhibited the growth of SGC-7901 cell-induced tumor in vivo by increasing the expression levels of pro-apoptotic proteins (Bax, c-caspase-3 and c-caspase-9) and reducing the expression levels of anti-apoptotic proteins (Survivin and Bcl-2) without adverse effects on the increasing body weight of nude mice. In conclusion, the present study revealed that isoimperatorin may be able to induce the apoptosis of SGC-7901 cells in vitro and in vivo by regulating the expression levels of mitochondria-mediated apoptosis-associated proteins.

\section{Introduction}

In previous decades, cancer has become a common disease in humans $(1,2)$. Currently, cancer is the second leading contributor to mortality rates in developing countries and the leading contributor to mortality rates in developed countries. In addition, with population growth and aging, and an unhealthy lifestyle comprising physical inactivity, smoking and 'westernized' diets, the burden of cancer is increasing in developing countries (3-6). Fortunately, various therapies, including surgical management (7), radiotherapy (8), chemotherapy (9), traditional Chinese medicine therapy (10), biotherapy (11), immunotherapy (12), gene therapy (13), thermotherapy (14), photodynamic therapy (15) and interventional therapy (16), have been successfully used to reduce pain in patients with cancer and prolong life expectancy.

Chemotherapy occupies an important position in cancer treatment, and it is reported that several components, including vinca alkaloids, taxanes, camptothecins and epipodophyllotoxins (17) exhibit potential antitumor activities. It is important to develop chemotherapy through the identification of plant-derived components with antitumor activity. Isoimperatorin (Fig. 1), a plant-derived component, exists widely in the Umbelliferae, and includes Angelica dahurica, Notopterygium incisum, Angelica pubescens and Peucedanum praeruptorum. Isoimperatorin has analgesic (18), antimicrobial (19), vascular relaxing (20) and anticancer activities (21-23). However, studies by Okuyama et al (21), Zhang (22) and Kim et al (23) focused predominantly on investigating the antiproliferative activity of isoimperatorin against different cancer cell lines using MTT or sulforhodamine B techniques. In the present study, the effects and possible mechanisms of isoimperatorin on SGC-7901 cells were examined in vitro, and SGC-7901 cell-induced tumors in vivo were 
examined by determining the inhibition rate, apoptotic rate, mitochondria-mediated apoptosis-associated proteins, tumor volumes and body weights of nude mice using MTT, flow cytometry, western blot analysis and xenograft assays.

\section{Materials and methods}

Chemicals and reagents. Isoimperatorin was obtained from the National Institutes for Food and Drug Control (Beijing, China) and was dissolved and diluted in 0.5\% DMSO to obtain different concentrations for the subsequent assays. RPMI 1640 media and fetal bovine serum (FBS) were purchased from Invitrogen (Thermo Fisher Scientific, Inc, Waltham, MA, USA). The MTT cell proliferation and cytotoxicity assay kit and Annexin V-fluorescein isothiocyanate (FITC)/propidium iodide (PI) apoptosis detection kit were purchased from Beyotime Institute of Biotechnology (Shanghai, China) and Yeasen (Shanghai, China). Primary antibodies for $\beta$-actin (catalog no. sc-47778), Survivin (catalog no. sc-8808), myeloid leukemia cell-1 (Mcl-1) (catalog no. sc-12756), B cell lymphoma-extra large (Bcl-xl) (catalog no. sc-8392), B cell lymphoma-2 (Bcl-2) (catalog no. sc-7382), second mitochondria-derived activator of caspase (Smac) (catalog no. sc-393118), Bcl-2-associated X factor (Bax) (catalog no. sc-7480), cleaved (c)-caspase-3 (catalog no. AC033) and horseradish peroxidase (HRP)-conjugated secondary antibodies (donkey anti-goat, catalog no. A0181; goat anti-mouse, catalog no. A0216; and goat anti-rabbit, catalog no. A0208) were purchased from were purchased from Beyotime Institute of Biotechnology, while primary antibody for c-caspase-9 (catalog no. 9501) was purchased from Cell Signaling Technology, Inc. (Danvers, MA, USA). All other chemicals and reagents used in the present study were analytical grade reagents.

Animals. Sixteen female nude mice (5-6 weeks old) were purchased from the SLRC Laboratory Animal Company (Shanghai, China) and housed in a temperature-controlled vivarium $\left(25^{\circ} \mathrm{C}\right)$ with a relative humidity of $65 \%$ and a $12 / 12$-h light-dark cycle. All mice had free access to water and food. All protocols for treatment of the animals were performed in strict accordance with the international ethical guidelines and the National Institutes of Health Guide concerning the Care and Use of Laboratory Animals (24). The experiments were performed with the approval of the Animal Experimentation Ethics Committee of Yinzhou People's Hospital, School of Medicine, Ningbo University (Ningbo, China; protocol no. 2013C50026AEEC).

Cell culture. The SGC-7901 cells were purchased from American Type Culture Collection (Manassas, VA, USA) and cultured in RPMI-1640 medium supplemented with 10\% FBS and antibiotics $(100 \mathrm{U} / \mathrm{ml}$ streptomycin and $100 \mathrm{U} / \mathrm{ml}$ penicillin) at $37^{\circ} \mathrm{C}$ in $5 \% \mathrm{CO}_{2} / 95 \%$ air. When the SGC-7901 cells reached logarithmic growth phase, the cells were subcultured and the experiments were performed immediately on the subcultured cells.

MTT reduction assay. The antiproliferative activity of isoimperatorin against SGC-7901 cells was evaluated using an MTT reduction assay. The SGC-7901 cells were seeded on 96-well culture plates with RPMI-1640 medium at a density of $5 \times 10^{3}$ cells/well. After $24 \mathrm{~h}$ of incubation at $37^{\circ} \mathrm{C}$ in $5 \%$ $\mathrm{CO}_{2} / 95 \%$ air, the cells were treated at different concentrations of isoimperatorin $(5,10,15,20,25,30,35$ and $40 \mu \mathrm{g} / \mathrm{ml})$ and $0.05 \%$ DMSO (control) for $48 \mathrm{~h}$ to examine dose-dependency; or with isoimperatorin at a concentration of $20 \mu \mathrm{g} / \mathrm{ml}$ and $0.05 \%$ DMSO (control) for 12, 24, 36, 48, 60 and $72 \mathrm{~h}$ to examine time-dependent effects. Subsequently, $20 \mu 1$ MTT $(5 \mathrm{mg} / \mathrm{ml})$ was separately added into each well, and the cells were cultured at $37^{\circ} \mathrm{C}$ in $5 \% \mathrm{CO}_{2} / 95 \%$ air for another $3 \mathrm{~h}$. Subsequently, $200 \mu \mathrm{l}$ DMSO was separately added into each well and the optical density (OD) of the DMSO solution was measured at $570 \mathrm{~nm}$ using a microplate reader (Thermo Fisher Scientific, Inc.). The inhibition rate of isoimperatorin against the SGC-7901 cells was determined using the following equation: Inhibition rate $(\%)=\left(\mathrm{OD}_{\text {control }}-\mathrm{OD}_{\text {treatment }}\right) / \mathrm{OD}_{\text {con- }}$ trol $\mathrm{X} 100$.

Apoptosis assay. Following treatment with isoimperatorin (5, 10 and $20 \mu \mathrm{g} / \mathrm{ml}$ ) and $0.05 \%$ DMSO (control) for $48 \mathrm{~h}$, the SGC-7901 cells were collected and washed three times with phosphate buffered-saline (PBS). Subsequently, the washed SGC-7901 cells $\left(5 \times 10^{3}\right.$ cells) were resuspended in $200 \mu \mathrm{l}$ staining buffer and stained with $10 \mu 1$ Annexin V-FITC (20 $\mu \mathrm{g} /$ $\mathrm{ml})$ and $5 \mu \mathrm{l} \mathrm{PI}(50 \mu \mathrm{g} / \mathrm{ml})$, following which, the SGC-7901 cells were quantified using flow cytometry and analyzed using CellQuest Pro 4.0 acquisition software (FACS Calibur; BD Biosciences, San Jose, CA, USA).

Western blot analysis. Following treatment with isoimperatorin $(5,10$ and $20 \mu \mathrm{g} / \mathrm{ml})$ and $0.05 \%$ DMSO (control) for $48 \mathrm{~h}$, the total proteins of the SGC-7901 cells were extracted with cell lysis buffer (catalog no. P0013; Beyotime Institute of Biotechnology), ultrasound and centrifugation at 12,000 x $g$ for $15 \mathrm{~min}$ at $4^{\circ} \mathrm{C}$. Protein concentration was determined using an Enhanced BCA Protein Assay kit (Applygen Technologies, Inc., Beijing, China). Then total proteins $(40 \mu \mathrm{g})$ were separated using $12 \%$ sodium dodecyl sulfate-polyacrylamide gel electrophoresis and transferred onto a PVDF membrane. Following blocking with $5 \%$ fat-free milk, the membrane was incubated with the anti- $\beta$-actin (monoclonal, mouse anti-human, 1:1,000), anti-Survivin (polyclonal, goat anti-human, 1:1,000), antiMcl-1 (monoclonal, mouse anti-human, 1:1,000), anti-Bcl-xl (monoclonal, mouse anti-human, 1:1,000), anti-Bcl-2 (monoclonal, mouse anti-human, 1:1,000), anti-Smac (monoclonal, mouse anti-human, 1:1,000), anti-Bax (monoclonal, mouse anti-human, 1:1,000), anti-c-caspase-3 (monoclonal, rabbit anti-human, 1:1,000) and anti-c-caspase-9 (monoclonal, rabbit anti-human, 1:1,000) primary antibodies overnight at $4^{\circ} \mathrm{C}$. The membrane was then washed with TBS-Tween (TBST-T) and incubated with the corresponding HRP-conjugated secondary antibody (monoclonal, donkey anti-goat, goat anti-mouse or goat anti-rabbit, 1:1,000) in TBS-T for $1 \mathrm{~h}$ at room temperature. Following another rinse, all proteins were detected using chemiluminescence (Beyo ECL Plus reagent; Beyotime Institute of Biotechnology). In order to assess protein loading, $\beta$-actin was selected as an internal control.

Antitumor activity of isoimperatorin in vivo. The SGC-7901 cells $\left(2 \times 10^{6}\right.$ cells per nude mouse) were subcutaneously 


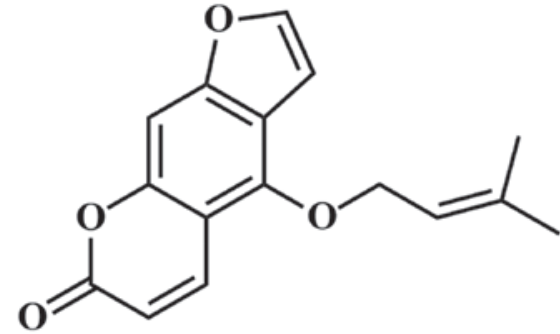

Figure 1. Chemical structure of isoimperatorin.

injected into the right flank of nude mice to establish the tumor xenograft model. When the SGC-7901 cells-induced tumors grew to 2-3 $\mathrm{mm}$ in diameter, the nude mice were randomly divided into control and isoimperatorin groups $(n=8)$ Animals in the control group received an intraperitoneal injection of $0.5 \% \mathrm{DMSO}$ and animals in the isoimperatorin group received an intraperitoneal injection of $10 \mathrm{mg} / \mathrm{kg}$; the injections were performed once each day for 20 days. The tumor length and width, and the body weight of the nude mice were measured on days $0,5,10,15$ and 20 using vernier calipers and electronic scales, and the tumor volumes were calculated using the following formula: Tumor volume $\left(\mathrm{mm}^{2}\right)=0.52 \mathrm{x}$ length $(\mathrm{mm}) \times$ width $^{2}(25)$. Finally, the nude mice were sacrificed immediately by decapitation, and then their tumor tissues were removed and collected for western blot analysis.

Statistical analysis. All data are presented as the mean \pm standard deviation. One-way analysis of variance (Dunnett test) was used to analyze the differences between two groups with SPSS 22.0 (IBM SPSS, Armonk, NY, USA). P<0.05 was considered to indicate a statistically significant difference.

\section{Results}

Cytotoxicity activity of isoimperatorin against SGC-7901 cells. The antiproliferative effects of isoimperatorin on SGC-7901 cells were evaluated using an MTT reduction assay. Following treatment with isoimperatorin $(5,10,15,20,25,30$, 35 and $40 \mu \mathrm{g} / \mathrm{ml}$ ) for $48 \mathrm{~h}$, proliferation of the SGC-7901 cells was significantly inhibited in a dose-dependent manner and the $\mathrm{IC}_{50}$ value was $18.75 \mu \mathrm{g} / \mathrm{ml}$ (Fig. 2A). Following treatment with isoimperatorin $(20 \mu \mathrm{g} / \mathrm{ml})$ for $12,24,36,48,60$ and $72 \mathrm{~h}$, proliferation of the SGC-7901 cells was significantly inhibited, also in a time-dependent manner (Fig. 2B).

Apoptosis of SGC-7901 cells induced by isoimperatorin. The results of the MTT reduction assay suggested that isoimperatorin inhibited the proliferation of SGC-7901 cells. Subsequently, flow cytometric analysis was used to investigate whether the antiproliferative activity of isoimperatorin against SGC-7901 cells was associated with apoptosis. As shown in Fig. 3A-D, following treatment with isoimperatorin $(5,10$ and $20 \mu \mathrm{g} / \mathrm{ml})$ for $48 \mathrm{~h}$, apoptosis of the SGC-7901 cells was significantly increased $(\mathrm{P}<0.01$; Fig. 3E), compared with the control group. The results of the flow cytometric analysis suggested that the antiproliferative activity of isoimperatorin against SGC-7901 cells was associated with apoptosis.
A
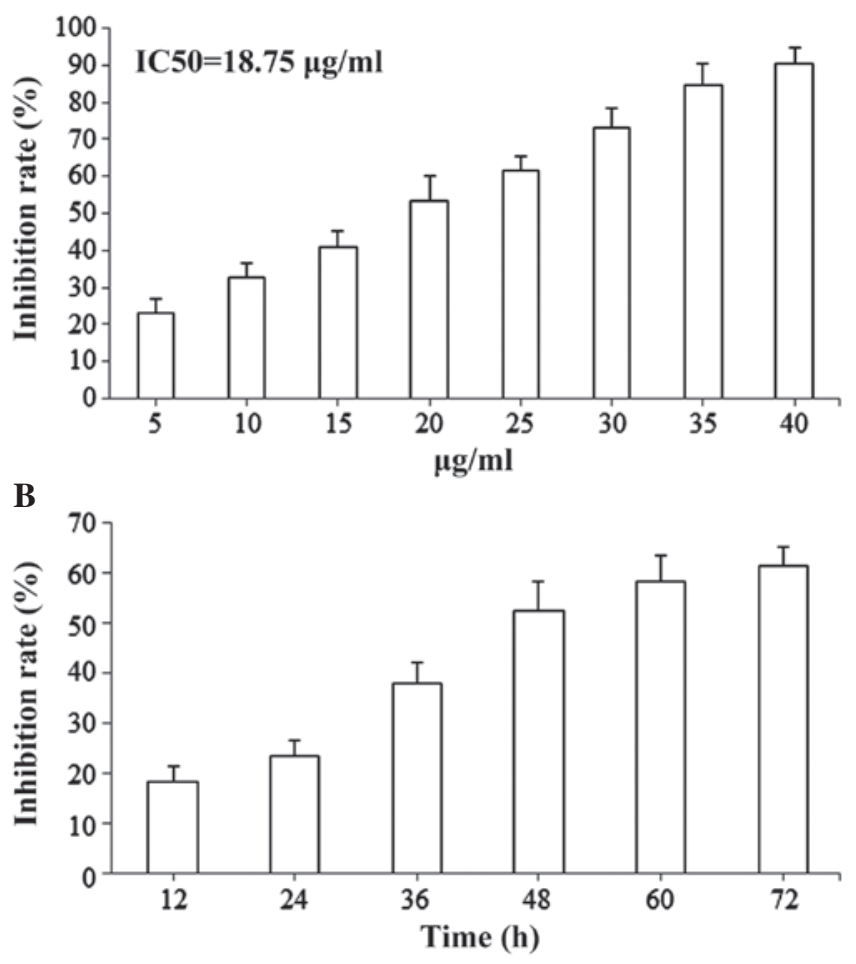

Figure 2. Cytotoxic activity of isoimperatorin against SGC-7901 cells. Changes occurred in a (A) dose-dependent and (B) time-dependent manner. $\mathrm{IC}_{50}$, half maximal inhibitory concentration.

Effects of isoimperatorin on the expression levels of mitochondria-mediated apoptosis-associated proteins. The results of the flow cytometric analysis indicated that isoimperatorin induced the apoptosis of SGC-7901 cells, therefore, western blot analysis was subsequently used to examine the pro-apoptotic mechanisms of isoimperatorin in the SGC-7901 cells. As shown in Fig. 4, following treatment with isoimperatorin $(5,10$ and $20 \mu \mathrm{g} / \mathrm{ml})$ for $48 \mathrm{~h}$, the protein expression levels of pro-apoptotic Bax, c-caspase-3 and c-caspase-9 were significantly $(\mathrm{P}<0.05$ or $\mathrm{P}<0.01)$ upregulated, and the protein expression levels of anti-apoptotic Survivin and Bcl-2 were significantly $(\mathrm{P}<0.01)$ downregulated, compared with the control group. No significant differences in the Mcl-1, Bcl-xl and Smac apoptosis-associated proteins were found.

Effects of isoimperatorin on SGC-7901 cells-induced xenograft model. The present study used the SGC-7901 cell-induced xenograft model to investigate the anti-gastric cancer activity of isoimperatorin in vivo. As shown in Fig. 5A, following treatment with isoimperatorin $(10 \mathrm{mg} / \mathrm{kg} /$ day for 20 days), the SGC-7901 cell-induced tumor growth in the nude mice was significantly inhibited $(\mathrm{P}<0.01)$, compared with that in the control group, whereas the increase in body weight of the nude mice was not significantly affected (Fig. 5B). Additionally, the results of the western blot analysis of tumor tissues (Fig. 6) suggested that, following treatment with isoimperatorin $(10 \mathrm{mg} / \mathrm{kg} /$ day for 20 days), the protein expression levels of pro-apoptotic Bax, c-caspase-3 and c-caspase-9 were significantly upregulated $(\mathrm{P}<0.01)$ and the protein expression levels of anti-apoptotic Survivin and Bcl-2 were significantly downregulated $(\mathrm{P}<0.01)$, compared with the control group. 

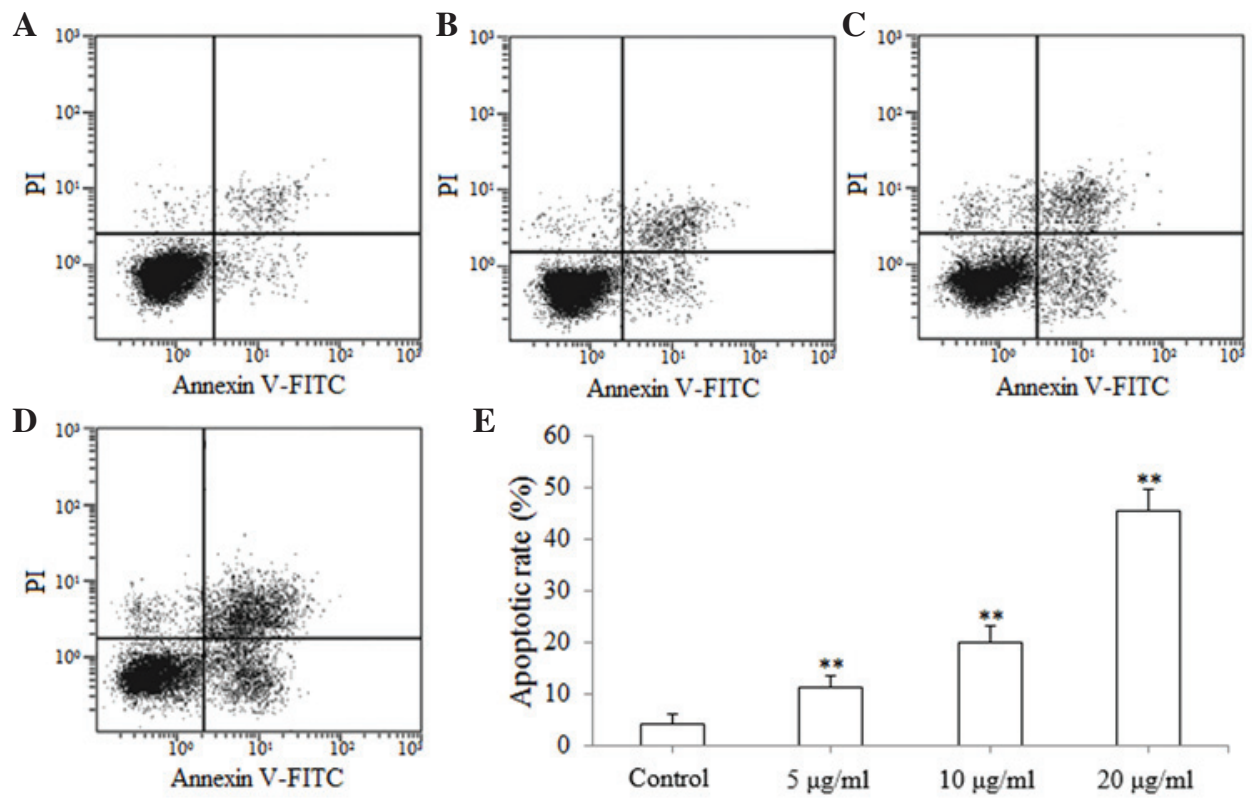

Figure 3. Apoptosis of SGC-7901 cells is induced by isoimperatorin. Results are shown for the (A) control, (B) $5 \mu \mathrm{g} / \mathrm{ml}$ isoimperatorin, (C) $10 \mu \mathrm{g} / \mathrm{ml}$ isoimperatorin and (D) $20 \mu \mathrm{g} / \mathrm{ml}$ isoimperatorin groups. (E) Graph showing the apoptotic rates. ${ }^{* *} \mathrm{P}<0.01$, compared with the control. FITC, fluorescein isothiocyanate; PI, propidium iodide.
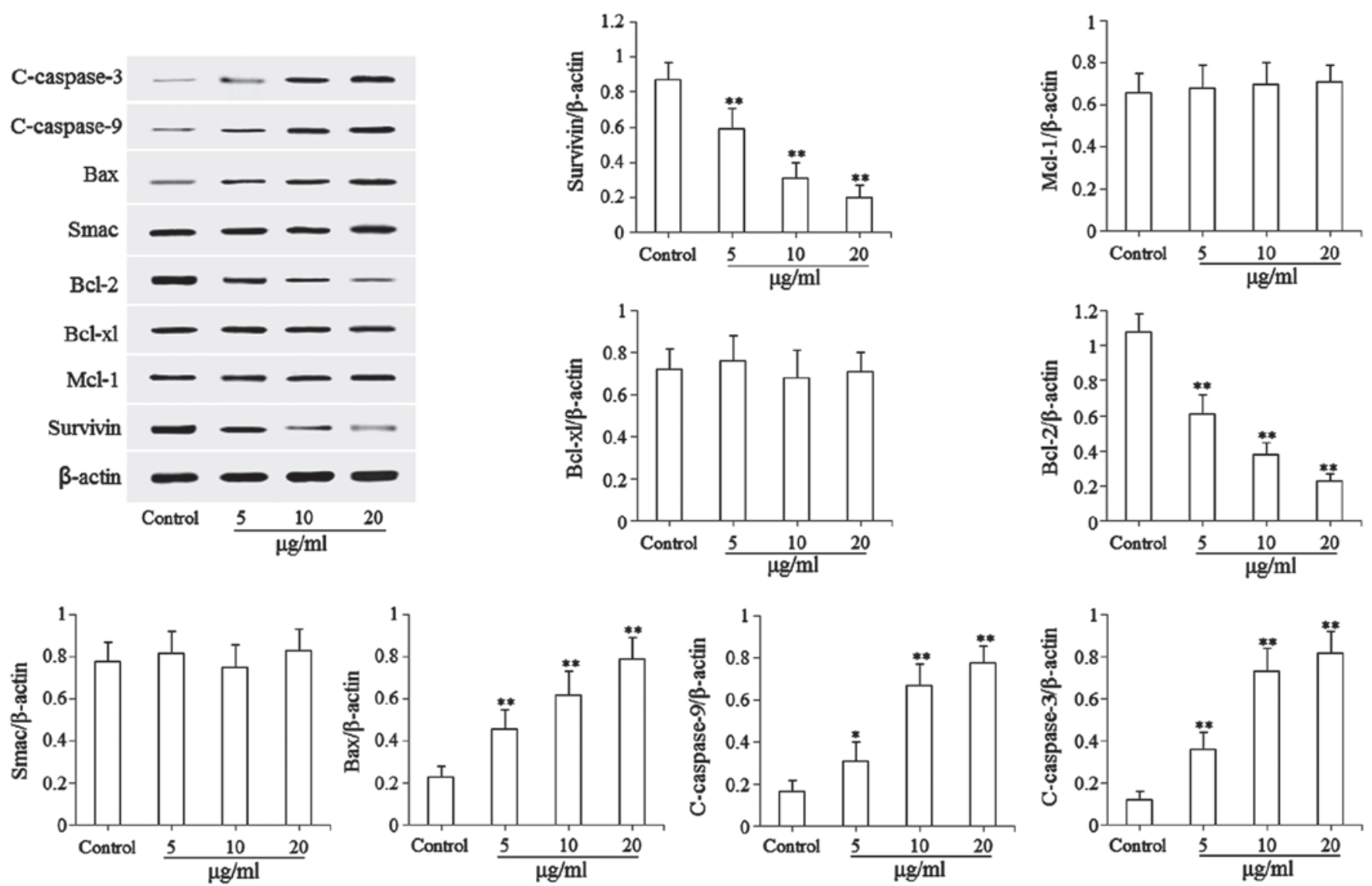

Figure 4. Effects of isoimperatorin on the expression levels of mitochondria-mediated apoptosis-associated proteins. Protein levels of Survivin, Mcl-1, Bcl-xl, Bcl-2, Smac, Bax, c-caspase-9 and c-caspase-3 were examined. " $\mathrm{P}<0.05$ and ${ }^{* * *} \mathrm{P}<0.01$, compared with the control. Mcl-1, myeloid leukemia cell-1; Bcl-2, B cell lymphoma-2; Bcl-xl, B cell lymphoma-extra large; Smac, second mitochondria-derived activator of caspase; Bax, Bcl-2-associated X factor.

\section{Discussion}

Isoimperatorin has been reported to have anticancer activity (21-23) and, although previous studies have focused predominantly on the antiproliferative activities of isoimperatorin against different cancer cell lines, the antiproliferative 
A

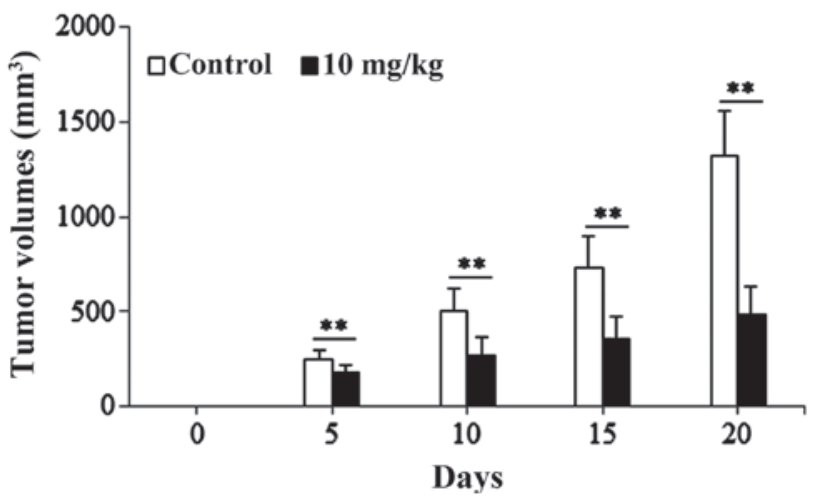

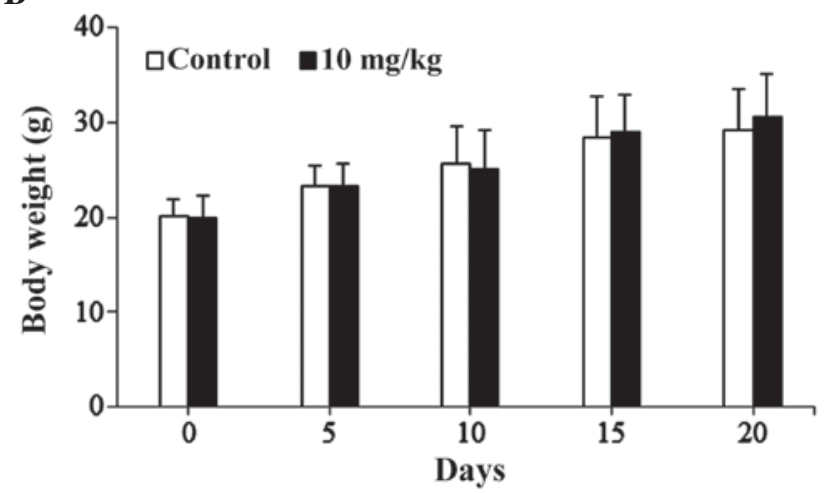

Figure 5. Effects of isoimperatorin on tumor volumes and body weights of nude mice in the SGC-7901 cell-induced xenograft model. (A) Tumor volume and (B) body weight changes are shown. ${ }^{* *} \mathrm{P}<0.01$, compared with the control.
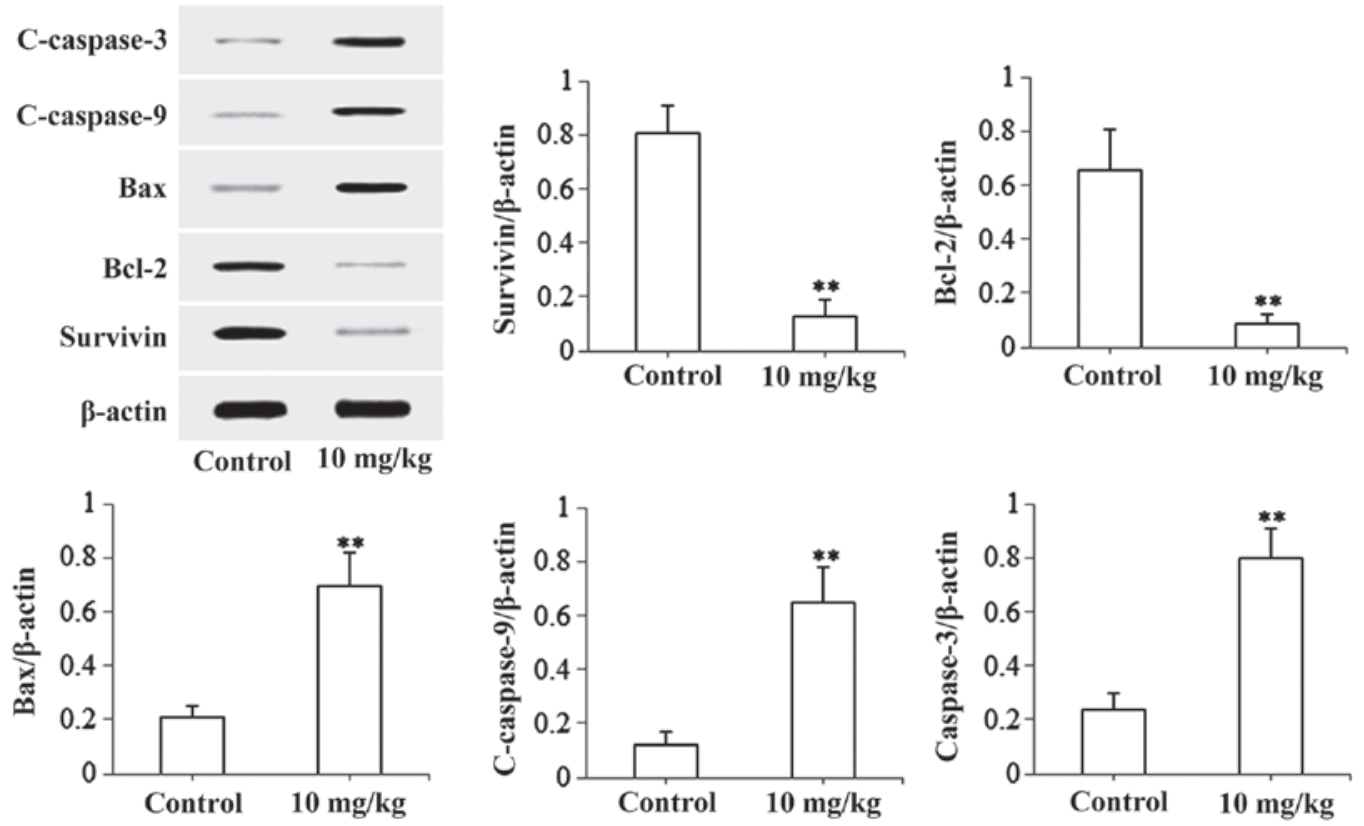

Figure 6. Effects of isoimperatorin on the expression levels of apoptotic protein. Protein expression levels of Survivin, Bcl-2, Bax, c-caspase-9 and c-caspase-3 were examined in the SGC-7901 cell-induced xenograft model. ${ }^{* *} \mathrm{P}<0.01$, compared with the control. Bcl-2, B cell lymphoma-2; Bcl-xl, B cell lymphoma-extra large; Smac, second mitochondria-derived activator of caspase; Bax, Bcl-2-associated X factor.

activities of isoimperatorin against SGC-7901 cells and the possible underlying mechanisms remain to be elucidated. Therefore, the present study investigated the antiproliferative activity of isoimperatorin against SGC-7901 cells and the possible underlying mechanisms using an MTT assay, flow cytometry, western blot analysis and xenograft assays. The results of the present study provided the first evidence, to the best of our knowledge, that isoimperatorin inhibited the proliferation of SGC-7901 cells by inducing apoptosis via the mitochondria-mediated pathway.

MTT assays are a commonly used method to examine the effects of anticancer agents on the proliferation of cancer cells $(26,27)$. In the present study, the results (Fig. 2A and B) of the MTT assay indicated that isoimperatorin significantly inhibited the proliferation of SGC-7901 cells in a dose- and time-dependent manner. Flow cytometric analysis is a common method used to investigate whether drugs can induce the apoptosis of cancer cells $(28,29)$. In the present study, the results of the flow cytometric analysis (Fig. 3) indicated that isoimperatorin significantly induced apoptosis of the SGC-7901 cells. The results of the MTT assay and flow cytometry indicated that the antiproliferative effect of isoimperatorin on SGC-7901 cells was associated with apoptosis.

Mitochondria-mediated apoptosis is an important pathway in the induction of cancer cell apoptosis. The apoptosis-associated proteins, including Bcl-2, Bcl-xl, Mcl-1, Survivin, Bax, Smac, caspase-3 and caspase-9, are important in the mitochondria-mediated apoptotic pathway $(30,31)$. When mitochondria are stimulated by apoptotic signals induced by anticancer agents, the expression levels and activation of the apoptosis-associated proteins are regulated. The associations among these apoptosis-associated proteins are complex in the mitochondria-mediated apoptotic pathway $(30,31)$. Firstly, the apoptotic signals stimulate the 
expression and release of Smac and cytochrome $c$ from the mitochondria to the cytoplasm, however, their release is inhibited by Bcl-2, Bcl-xl and Mcl-1, whereas Bax inhibits the function of Bcl-2, Bcl-xl and Mcl-1 (32). In the present study, isoimperatorin significantly upregulated the expression level of Bax and downregulated the expression level of Bcl-2, without affecting the expression levels of Bcl-xl and $\mathrm{Mcl}-1$, indicating that isoimperatorin promoted the release of cytochrome $c$ from the mitochondria to the cytoplasm. The released cytochrome $c$ promotes the activation of cytochrome $c$-dependent caspase- 9 and caspase- 3 to generate c-caspase-9 and c-caspase-3 (33). In the present study, isoimperatorin significantly upregulated the expression levels of c-caspase-9 and c-caspase-3, indicating that isoimperatorin promoted the activation of caspase- 9 and caspase-3. The activation of caspase- 9 and of caspase- 3 are inhibited by the inhibitor of apoptosis protein (IAP), Survivin; however, Smac eliminates IAP-induced inhibition (34). In the present study, isoimperatorin significantly downregulated the expression level of Survivin without affecting the expression level of Smac, suggesting that isoimperatorin eliminated IAP-induced inhibition to promote the activation of caspase- 9 and caspase- 3 . The apoptosis of cancer cells is induced by effective caspases (c-caspase-3). The results of the western blot analysis (Fig. 4) suggested that isoimperatorin significantly induced the apoptosis of SGC-7901 cells in vitro by regulating the expression levels of mitochondria-mediated apoptosis-associated proteins. In addition, the results of the xenograft assay (Figs. 5 and 6) indicated that isoimperatorin exhibited a significant inhibitory effect on SGC-7901 cell-induced tumor growth without adversely affecting the body weight increase of nude mice in vivo by regulating the expression levels of mitochondria-mediated apoptosis-associated proteins.

In conclusion, the present study revealed that isoimperatorin may be able to induce the apoptosis of SGC-7901 cells in vitro and in vivo by regulating the expression levels of mitochondria-mediated apoptosis-associated proteins. However, further investigations are required to confirm the pro-apoptotic activity and mechanisms of isoimperatorin on SGC-7901 cells.

\section{Acknowledgements}

This study was supported by The Science and Technology Planning Project from Ningbo, China (grant no. 2013C50026).

\section{References}

1. Ries LAG, Melbert D, Krapcho M, Stinchcomb DG, Howlader N, Horner MJ, Mariotto A, Miller BA, Feuer EJ, Altekruse SF, et al: SEER cancer statistics review, 1975-2005. Bethesda MD National Cancer Institute 1975-2005, 2008 (https://hero.epa.gov/ hero/index.cfm/reference/details/reference_id/730406).

2. Jemal A, Bray F, Center MM, Ferlay J, Ward E and Forman D: Global cancer statistics. CA Cancer J Clin 61: 69-90, 2011.

3. Horton R: GBD 2010: Understanding disease, injury, and risk. Lancet 380: 2053-2054, 2012.

4. Yancik R and Ries LA: Cancer in older persons: An international issue in an aging world. Semin Oncol 31: 128-136, 2004.

5. Hu G, Tuomilehto J, Silventoinen K, Barengo NC, Peltonen M and Jousilahti P: The effects of physical activity and body mass index on cardiovascular, cancer and all-cause mortality among 47212 middle-aged Finnish men and women. Int J Obesity (Lond) 29: 894-902, 2005.
6. Moreira DM, Aronson WJ, Terris MK, Kane CJ, Amling CL, Cooperberg MR, Boffetta P and Freedland SJ: Cigarette smoking is associated with an in increased risk of biochemical disease recurrence, metastasis, castration-resistant prostate cancer, and mortality after radical prostatectomy: Results from the SEARCH database. Cancer 120: 197-204, 2014.

7. Speer AG, Thursfield VJ, Torn-Broers $Y$ and Jefford M: Pancreatic cancer: Surgical management and outcomes after 6 years of follow-up. Med J Aust 196: 511-515, 2012.

8. Jia L, Ma S, Hou X, Wang X, Qased AB, Sun X, Liang N, Li H, Yi H, Kong D, et al: The synergistic effects of traditional Chinese herbs and radiotherapy for cancer treatment. Oncol Lett 5: 1439-1447, 2013

9. Romiti A, Cox MC, Sarcina I, Di Rocco R, D'Antonio C, Barucca V and Marchetti P: Metronomic chemotherapy for cancer treatment: A decade of clinical studies. Cancer Chemother Pharmacol 72: 13-33, 2013.

10. Li X, Yang G, Li X, Zhang Y, Yang J, Chang J, Sun X, Zhou X, Guo Y, Xu Y, et al: Traditional Chinese medicine in cancer care: A review of controlled clinical studies published in Chinese. PLoS One 8: e60338, 2013.

11. Gopalakrishnan G, Lepetre S, Maksimenko A, Mura S, Desmaële D and Couvreur P: Lipid-conjugation of endogenous neuropeptides: Improved biotherapy against human pancreatic cancer. Adv Healthc Mater 4: 1015-1022, 2015.

12. Vanneman M and Dranoff G: Combing immunotherapy and target therapies in cancer treatment. Nat Rev Cancer 12: 237-251, 2012.

13. Duarte S, Carle G, Faneca H, de Lima MC and Pierrefite-Carle V: Suicide gene therapy in cancer: Where do we stand now? Cancer Lett 324: 160-170, 2012.

14. Vrbova B and Vrba J: Microwave thermotherapy in cancer treatment: Evaluation of homogeneity of SAR distribution. Prog Electromagn Res 129: 181-195, 2012.

15. Brown SB, Brown EA and Walker I: The present and future role of photodynamic therapy in cancer treatment. Lancet Oncol 5: 497-508, 2004.

16. Niu HX, Du T, Xu ZF, Zhang XK and Wang RG: Role of wild type p53 and double suicide genes in interventional therapy of liver cancer in rabbits. Acta Cir Bras 27: 522-528, 2012.

17. Nobili S, Lippi D, Witort E, Donnini M, Bausi L, Mini E and Capaccioli S: Natural compounds for cancer treatment and prevention. Pharmacol Res 59: 365-378, 2009.

18. Wang MY, Jia MR, Ma YY and Li XB: Pharmacological effect of four linear furocoumarins in radix Angelicae dahuricae. Nat Prod Res Dev 22: 485-489, 2010.

19. Kwon YS, Kobayashi A, Kajiyama S, Kawazu K, Kanzaki H and Kim CM: Antimicrobial constituents of Angelica dahurica roots. Phytochemistry 44: 887-889, 1997.

20. Li ZK, Liu MQ and Yang HJ: The study of vasoactive of pungent herbs' chemical constituents in Umbelliferae. Pharmacol Clin Chin Mat Med 25: 38-40, 2009.

21. Okuyama T, Takata M, Nishino H, Nishino A, Takayasu J and Lwashima A: Studies on the antitumor-promoting activity of naturally occurring substances. II. Inhibition of tumor-promoter-enhanced phospholipid metabolism by umbelliferous materials. Chem Pharm Bull (Tokyo) 38: 1084-1086, 1990.

22. Zhang XX: Studies on the antitumor constituents of Notopterygium incisum. [dissertation]. Hebei Med Univ 1-117, 2009.

23. Kim YK, Kim YS and Ryu SY: Antiproliferative effect of furanocoumarins from the root of Angelica dahurica on cultured human tumor cell lines. Phytother Res 21: 288-290, 2007.

24. National Research Council (US) Committee for the Update of the Guide for the Care and Use of Laboratory Animals: Guide for the Care and Use of Laboratory Animals, 8th edition. National Academies Press (US), Washington, DC, 2011.

25. Zhao YL, Zhao LJ, Luo YX, Li X, Zhang YF, Liu XD, Luo YG and Zhong LL: Synergistic effect of radiation and traditional Chinese medicine Rhizoma Typhonii ethanol extract depends on p53 expression in treatment of lewis mouse lung cancer cells. Afr J Tradit Complement Altern Med 12: 109-114, 2015.

26. Acquaviva R, Di Giacomo C, Sorrenti V, Galvano F, Santangelo R, Cardile V, Gangia S, D'Orazio N, Abraham NG and Vanella L: Antiproliferation effect of oleuropein in prostate cell lines. Int J Oncol 41: 31-38, 2012.

27. Lee J, Gupta S, Huang JS, Jayathilaka LP and Lee BS: HPLC-MTT assay: Anticancer activity of aqueous garlic extract is from allicin. Anal Biochem 436: 187-189, 2013. 
28. Li W, Li DY, Wang HD, Zheng ZJ, Hu J and Li ZZ: Juglans regis hexane extract exerts antitumor effect, apoptosis induction and cell circle arrest in prostate cancer cells in vitro. Trop J Pharm Res 14: 399-405, 2015.

29. Zi FM, He JS, Li Y, Wu C, Yang L, Yang Y, Wang LJ, He DH, Zhao Y, Wu WJ, et al: Metformin displays anti-myeloma activity and synergistic effect with dexamethasone in in vitro and in vivo xenograft models. Cancer Lett 356: 443-453, 2015.

30. Shi Y: A structure view of mitochondria-medated apoptosis. Nat Struct Biol 8: 394-401, 2001.

31. Wang X: The expanding role of mitochondria in apoptosis. Genes Dev 15: 2922-2933, 2001.
32. Yang XK, Xu MY, Xu GS, Zhang YL and Xu ZX: In vitro and in vivo antitumor activity of scutebarbatine $A$ on human lung carcinoma A549 lines. Molecules 19: 8740-8751, 2014.

33. Kim J, Parrish AB, Kurokawa M, Matsuura K, Freel CD, Andersen JL, Johnson CE and Kornbluth S: Rsk-mediated phosphorylation and 14-3-3e binding of Apaf-1 suppresses cytochrome c-induced apoptosis. EMBO J 31: 1279-1292, 2012.

34. Du C, Fang M, Li Y, Li L and Wang X: Smac, a mitochondrial protein that promotes cytochrome c-dependent caspase activation by eliminating IAP inhibition. Cell 102: 33-42, 2000. 